\title{
CORONAL MAGNETIC FIELDS DEDUCED FROM RADIO METHODS
}

\author{
A. KRÜGER AND J. HILDEBRANDT \\ Astrophysical Institute Potsdam, An der Sternwarte 16, \\ D-0-1591 Potsdam-Babelsberg, Germany
}

\begin{abstract}
Major problems of the physics of the solar atmosphere and processes of solar activity are due to the poor knowledge of the magnetic fields outside the photosphere. Unique methods to determine magnetic fields in the corona and chromosphere make use of radio observations in close connection with information obtained in other spectral ranges, e.g. the optical and X-ray regions. Based on relevant emission and propagation processes, the basic radio methods providing information on the parent magnetic fields are summarized. Signatures in the microwave and meter wave regions are used to derive magnetic field parameters at different levels in the solar atmosphere of active regions during quiet and flaring conditions. Implications on fine and gross structures are briefly discussed and consequences on acting physical processes mentioned.
\end{abstract}

\section{INTRODUCTION}

The solution of open questions about the physics of the solar atmosphere suffers from the difficulty of measuring magnetic fields of the Sun outside the photosphere. Although qualitative evidence for magnetic fields in the chromosphere and corona can be found in observations in different spectral regions from X-rays to optical waves, radio waves are favored to produce quantitative information on magnetic fields. The radio methods, however, in contrast to the Zeeman effect of optical waves in the photosphere, mostly concern indirect methods of determination of magnetic fields and typically require additional information or working hypotheses.

Estimates of an average coronal magnetic field distribution have been reviewed by cf. Newkirk (1967); and Dulk \& McLean (1978). New information is available (and still more is needed!) which probably applies to fine structures of the magnetic field. The present contribution aims to discuss basic diagnostic tools offered by radio and their application to the determination of coronal magnetic fields in undisturbed and disturbed (flaring) parts of solar active regions. 


\section{MICROWAVE DIAGNOSTICS}

\section{Gyromagnetic radiation}

Gyromagnetic radiation at low harmonics $(s \lesssim 4)$ of the gyrofrequency $\nu_{B}$ is responsible for the S-component emission at $\mathrm{cm}$ - and lower $\mathrm{dm}$-waves and therefore, can be well used to explore magnetic fields of solar active regions. Advanced emission models allowing diagnostics of the magnetic field have been treated by several authors (Gelfreikh \& Lubyshev 1979; Alissandrakis et al. 1980; Krüger et al. 1985; Holman \& Kundu 1985). These models reveal two important aspects:

1) Since the absorption coefficient determining the spectrum and polarization of the gyromagnetic radiation depends on the strength and orientation of the magnetic field, spatially resolved information at different test frequencies is required.

2) Since the absorption coefficient also depends on other parameters like electron density (plasma frequency) and energy (temperature), an accurate magnetic field diagnostic method has to presume the knowledge of these quantities from additional measurements and model assumptions.

Taking into account these circumstances, the magnetic field can be derived by finding the best fit between the observed quantities and the models. Typically the following observed quantities have been used:

- spatial distribution of the Stokes vectors I and V at selected frequencies,

- the frequency spectrum of $I$ and $V$,

- the limiting frequency e.g. of the third gyroharmonic $\nu_{B 3}$ referring to the lower boundary of the corona, where the magnetic field $B$ is given by $B=0.12 \nu_{B 3}\left[\mathrm{MHz}_{\mathrm{z}}[G]\right.$,

- diameters of S-component sources above sunspots and their dependence on frequency. (For results cf. Section 4).

Gyro-synchrotron radiation

Gyromagnetic radiation at higher harmonics corresponds to greater energy of the emitting electrons (called gyro-synchrotron radiation) and has been applied to radio burst emission. The information about the magnetic field can be extracted from the shape (frequency extent) and position (spectral maximum) of the emitted spectrum.

If the density of the ambient medium increases so that the influence of the refractive index cannot be neglected, one obtains the well-known Razin effect where a cutoff is present at frequencies $\nu \leq \nu_{R}=$ const $N_{e} /(B \sin \phi)$ ( $N_{e}$ - electron density, $\phi$ - pitch angle). 
Coulomb bremsstrahlung

Coulomb bremsstrahlung is the second important emission mechanism related to solar microwave emission. Model calculations considering this mechanism have been performed and used by Alissandrakis et al. (1980), Krüger et al. (1985), Urpo et al. (1987), Brosius \& Holman (1988), and others. Since the bremsstrahlung process is not primarily related to magnetic fields, the influence of magnetic fields on the emission comes through the anisotropic refractive index. Hence, possibilities of a magnetic field diagnostic appear as follows:

- In the case of optically thin bremsstrahlung under the condition of quasilongitudinal wave propagation the degree of polarization is given by

$$
p=\frac{2 Y_{L}}{1+Y_{L}^{2}}, \quad\left(Y_{L}=\frac{\nu_{B}}{\nu} \cos \theta\right)
$$

( $\theta=$ angle between the magnetic field and the wave vector).

- In the case of optically thick bremsstrahlung and inhomogeneous media, the degree of polarization differs from zero. In the presence of a gradient of the temperature the degree of polarization becomes

$$
p=\frac{\partial\left(\ln T_{b}\right)}{\partial(\ln \nu)} \frac{\nu_{B}}{\nu}|\cos \theta|
$$

( $T_{b}$ - brightness temperature; cf. Gelfreikh 1990).

\section{Wave propagation}

A number of wave propagation effects apply to the diagnostics of coronal magnetic fields, e.g. the reversal of polarization in a region of quasi-transverse propagation (QT-region), the Faraday effect, and the occurrence of ordinary polarization in regions near the absorption edges of the refractive index.

The theory of electromagnetic mode coupling was extended by Cohen (1960) to waves traversing spatially-varying magnetic fields and applied to microwaves by Peterova \& Akhmedov (1973), Peterova \& Ryabov (1981), and Bandiera (1982). A special effect is of interest when the wave crosses a QT-region. Then one finds the condition that for frequencies $\nu^{4} \ll \nu_{t}^{4}$ weak coupling is present and a reversal of the sense of polarization takes place after the waves pass through that region.

The critical frequency $\nu_{t}$ is given by

$$
\nu_{t}=\text { const }\left[\frac{N_{e} B^{3}}{\frac{d \theta}{d z}}\right]^{1 / 4} \sim\left[\frac{\nu_{B}^{3} \nu_{p}^{2}}{S}\right]^{1 / 4}
$$

and

$$
B=\text { const } \frac{\nu_{t}^{4 / 3}}{\left(L_{B} N_{e}\right)^{1 / 3}} \quad, \quad S=\left(\frac{d \theta}{d z}\right) \approx L_{B}^{-1}
$$




\section{METER-WAVE DIAGNOSTICS}

Compared to the microwaves, the radio emission at longer wavelengths (abbreviated as "meter waves") covers a much greater range of coronal heights. Since the Quiet-Sun and S-component radiation at these wavelengths is rather weak, most of this emission and related methods of magnetic-field estimation refer to radio bursts.

\section{MHD shock waves and slow-drift bursts}

The frequency drift rates depending on the shock velocity and the phenomenon of band splitting of type II bursts have been used for a long time to derive information about magnetic fields. Difficulties arise from the fact that a uniform and generally accepted theory of type II bursts (as also for the most other types of meter-wave bursts) is still missing.

a) Shock velocity:

There are different approaches to explain how plasma waves are excited by shock waves associated with type II bursts. According to the earliest approach by Pikelner \& Gintsburg (1963) the Langmuir waves are excited by an MHD shock if the Alfvénic Mach number $M_{A}$ exceeds a critical Mach number $M_{c}$ given by

$$
M_{A}=\frac{v}{v_{A}}>M_{c}=1+\frac{3}{8}\left(\frac{8 \pi N k T}{B^{2}}\right)
$$

where

$$
\frac{B^{2}}{8 \pi} \gg N k T
$$

b) Band splitting:

The effect of band splitting of type II bursts can be interpreted in different ways either by magnetic or geometrical effects. If one looks for magnetic effects three variants of interpretation are to be noted:

- Emission separation by magneto-ionic resonances (Sturrock 1961)

$$
\nu_{1}=\nu_{p} ; \quad \nu_{2}=\nu_{u h}=\left(\nu_{p}^{2}+\nu_{B}^{2}\right)^{1 / 2} ; \Delta \nu \approx \frac{1}{2} \frac{\nu_{B}^{2}}{\nu_{p}}\left(\nu_{p} \gg \nu_{B}\right)
$$

( $\nu_{p}$ - plasma frequency, $\nu_{u h}$ - upper hybrid frequency).

- Emission separation by zeros of the refractive index (Roberts 1959)

$$
\nu_{1}=\nu_{p} ; \quad \nu_{2} \approx \nu_{p}+\frac{1}{2} \nu_{B} ; \quad \Delta \nu \approx \frac{1}{2} \nu_{B}\left(\nu_{p} \gg \nu_{B}\right) .
$$

- Another approach was proposed by Smerd, Sheridan \& Stewart (1975) by deriving the related Mach number $M$ from the Rankine-Hugoniot jump condition and hence concluding the magnetic field from the shock speed

$$
B=5.1 \cdot 10^{-5} v_{[k m / s]} \nu_{p[M H z]} M^{-1}[G]
$$

$\left(v\right.$ - shock speed, $\nu_{p}$ - plasma frequency ahead the shock front). 


\section{Fast-drift bursts}

a) Polarization of harmonic radiation

According to Melrose et al. (1978) the degree of polarization $p$ of the second harmonic of the plasma frequency is

$$
p=f\left(\theta, \theta_{o}\right) \frac{\nu_{B}}{\nu_{p}}
$$

where $f$ is a function of the angle $\theta$ between wave propagation and the magnetic field $B$, and $\theta_{o}$ denotes the limiting cone angle of the distribution of Langmuir waves.

b) Time profile of ordinary polarization

Due to wave propagation, the time profile of the degree of polarization of a fast-drift burst indicates the magnetic field. At the beginning of such bursts the totally polarized part corresponds to the distance $\Delta s$ between the escape levels of the ordinary and extraordinary wave modes so that

$$
\Delta s=v_{d} \Delta t=\frac{\nu_{B}}{\nu} L_{N}
$$

where $v_{d}$ is the drift velocity of the exciter and

$$
L_{N}=N_{e}\left(\frac{d N_{e}}{d s}\right)^{-1}
$$

is the scale height of the electron density $N_{e}$ (Fomichev \& Chertok 1965).

\section{Spectral fine structures}

Spectral fine structures are abundant in nearly all types of solar meter wave emission. Technical difficulties of the magnetic-field determination often arise from the lack of simultaneous spectral and spatial resolution. Examples of basic processes coming into consideration for magnetic field diagnostics are the following:

a) Double plasma resonance

The condition for double plasma resonance $\nu_{p} \approx s \nu_{B}$ was applied for the interpretation of fine structures in U-type bursts and zebra patterns (Zheleznyakov \& Zlotnik 1975; Kuijpers 1975).

b) Whistler waves

The drift rate of fiber bursts $D=d \nu / d t$ interpreted as the signature of whistler waves is given by

$$
D=\frac{\nu}{2 L_{N}} v_{w}=c \frac{\nu}{L_{N}}\left(\frac{\nu_{B}}{\nu_{p}}\right)\left(\frac{\nu_{w}}{\nu_{B}}\left(1-\frac{\nu_{w}}{\nu_{B}}\right)^{3}\right)^{1 / 2}
$$

where $v_{w}$ is the group velocity and $\nu_{w}$ the frequency of whistler waves, respectively (Mann \& Baumgärtl 1988). 


\section{MAGNETIC FIELDS}

Average distribution

Now we consider quantitative results of radio observations applied to the average height distribution of coronal magnetic fields. The most direct access is given by gyromagnetic microwave emission. Interpreting such observations made e.g. by the VLA or RATAN-600 (cf. Gelfreikh, these proceedings), a magnetic dipole field can be used as reference model is given by

$$
B_{z}=B_{m} z_{d}^{3}\left(z+z_{d}\right)^{-3}
$$

where $z_{d}$ is the assumed dipole depth below the photosphere. An analysis of 36 compact $\mathrm{S}$-component sources observed at RATAN-600 is consistent with an average magnetic field at the bottom of the corona $\left(z \approx 2 \cdot 10^{3} \mathrm{~km}\right)$ of $1750 \mathrm{G}$, a magnetic scale height

$$
L_{B}=B(z)(d B / d z)^{-1}
$$

of $7 \cdot 10^{3} \mathrm{~km}$, a gradient of the magnetic field

$$
d B_{z} / d z=-(0.25 \pm 0.1) \mathrm{G} / \mathrm{km}
$$

corresponding to a gradient of $0.37 \mathrm{G} / \mathrm{km}$ at the level of the photosphere (Krüger et al. 1986).

Confirming evidence of fields of $1800 \mathrm{G}$ existing in the corona above sunspots has been obtained recently by VLA observations (White et al. 1991).

As a reference, a dipole field with parameters $B_{m}=2500 \mathrm{G}$ and $z_{d}=2 \cdot 10^{4}$ $\mathrm{km}$ is given in Figure 1. The force-free extrapolated field (black dots in Figure 1) of a magnetogram measured at Potsdam on 4 July 1973 (Hildebrandt et al. 1984) shows an astonishing agreement with the dipole distribution. Up to a height of about $1.5 \cdot 10^{5} \mathrm{~km}$ above the photosphere, the same dipole distribution is in accordance with fields derived from the reversal of circular polarization in QT-regions marked in by crosses in Figure 1 (Peterova \& Ryabov 1981).

In contrast to the data quoted above, the magnetic fields derived from different burst observations can be different and partly exceed the maximum dipole field deduced from S-component observations. This is the case in particular for the fields obtained from the interpretation of fiber bursts by whistler waves (e.g., Mann \& Baumgärtl 1988) or from type II bursts (e.g., Fomichev \& Chertok 1965). Early radio observations cited by Newkirk (1967) and Dulk \& McLean (1978), e.g. of type I bursts, indicate even higher magnetic fields but suffer from uncertainties. Due to possible density irregularities, for other magnetic field estimations the absolute height scale is not very certain (e.g. Batchelor et al. 1984; Bruggmann et al. 1990; Lang et al. 1987). 


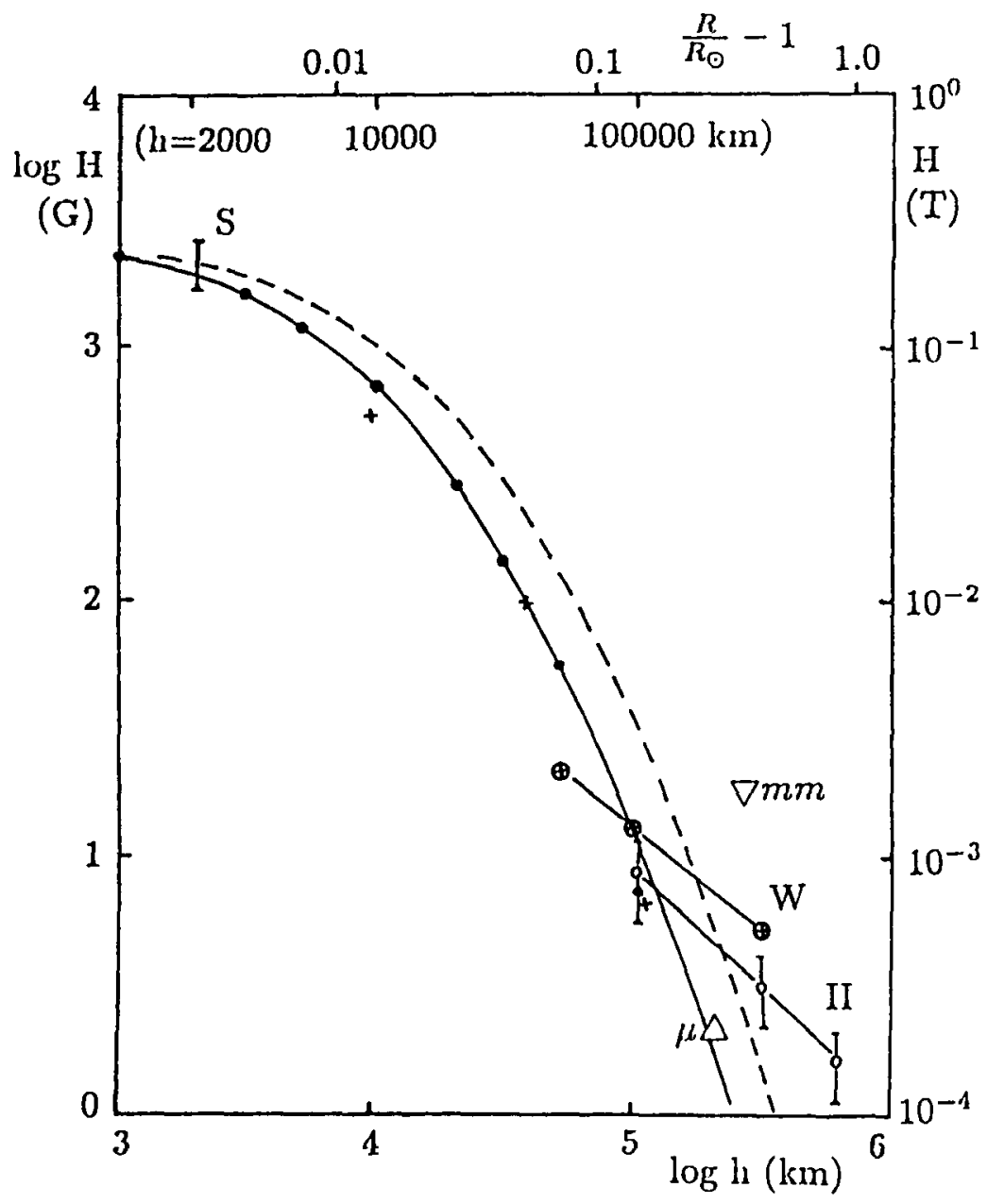

Fig. 1. Measures of magnetic fields compared with dipole distributions above the center of a large sunspot $\left(B_{m}=2500 \mathrm{G}\right.$, full line: dipole depth $z_{d}=2 \cdot 10^{4} \mathrm{~km}$, broken line: $z_{d}=3 \cdot 10^{4} \mathrm{~km}$ ); black dots refer to a force-free extrapolated photospheric field; $S$ - S-component, $W$ fiber bursts (whistler waves), II - type II bursts, $\mu$ - microwave burst (1984 February 16), mm-mm-burst (1989 September 9). 
Magnetic-field fine structures

The field distribution of Figure 1 can give only a general and crude information about the magnitude of magnetic fields in the corona averaged over a certain space volume. Besides, there is evidence of different kinds of fine structures below the present limits of angular resolution by filamentation and turbulence. (cf. Stenflo 1973; Hoyng 1980).

Deviations of the magnetic field from a force-free field may be indicated by $\mathrm{S}$-component observations. It is interesting that both dipole models and extrapolated sunspot fields yield ring-like emission patterns which, however, according to VLA observations are scarcely observed (e.g. Kundu \& Alissandrakis 1984). Internal turbulent fluctuations of the magnetic field causing variations of the magnetic field direction are considered to have an effect of smearing out the ring structures (Krüger \& Hildebrandt 1991).

\section{$\underline{\text { Limb events }}$}

The principal difficulty that radio data do not contain direct information about absolute height levels in the corona is overcome by limb observations. An extreme example of an off-limb source is provided by the behind-limb flare on 16 February 1984 (cf. Krüger et al. 1991). In this case $8 \mathrm{GHz}$-emission was detected at a height of $\geq 2 \cdot 10^{5} \mathrm{~km}$ above the photosphere (cf. Figure 1). A still more spectacular flare event occurred on 29 September 1989 where for the first time $87-\mathrm{GHz}$ radiation was recorded at presumably more than $3 \cdot 10^{5} \mathrm{~km}$ above the limb (cf. Krüger \& Urpo 1992).

\section{CONCLUSIONS}

Summarizing the present state of magnetic-field estimations by radio methods we obtain the following conclusions:

(1) At the bottom of the corona magnetic fields up to about $2 \mathrm{kG}$ are indicated by gyromagnetic radiation of the S-component. This is consistent with a dipole active-region (sunspot) reference field with scale lengths of the order $10^{4} \mathrm{~km}$ in the low corona under nonflaring conditions.

(2) Magnetic fields derived by various radio-burst emissions tend to exceed the dipole distribution where direct height estimations from off-limb sources support indirect methods depending on given electron density models. Magnetic-field transport by plasma flows may be responsible for a height dependence less than $H \sim R^{-3}$.

(3) Superimposed on the mean height distribution of magnetic fields, there is evidence for the existence of fine structures of the field for both flaring and nonflaring conditions.

(4) Magnetic fine structures are expected to be intimately related to fragmentary field dissipation (reconnection) leading to particle acceleration and plasma heating signified by radio emission.

\section{REFERENCES}

Alissandrakis, C.E., Kundu, M.R., \& Lantos, P. 1980, A\&A, 82, 30

Bandiera, R. 1982, A\&A, 112, 52 
Batchelor, D.A., Benz, A.O., \& Wiehl, H.J. 1984, ApJ, 280, 879

Brosius, J.W., \& Holman, G.D. 1988, ApJ, 327, 417

Bruggmann, G., Benz, A.O., Magun, A., \& Stehling, W. 1990, A\&A, 240, 506

Cohen, M.H. 1960, ApJ, 131, 664

Dulk, G.A., \& McLean, D.J. 1978, Solar Phys. 57, 279

Fomichev, V.V., \& Chertok, I.M. 1965, AZh, 42, 1256

Gelfreikh, G.B. 1990, Astron. Nachr., 311, 385

Gelfreikh, G.B., \& Lubyshev, B.I. 1979, AZh, 56, 562

Hildebrandt, J., Seehafer, N., \& Krüger, A. 1984, A\&A, 134, 185

Holman, G.D., \& Kundu, M.R. 1985, ApJ, 292, 291

Hoyng, P., Duijveman, A., van Grunsven, Th.F.J., \& Nicholson, D.R. 1980, in Solar and Interplanetary Dynamics, ed. M. Dryer \& E. TandbergHanssen (Dordrecht:D. Reidel), 299

Krüger, A. \& Hildebrandt, J. 1991, Contribution to the XXI General Assembly of IAU, Buenos Aires.

Krüger, A., Hildebrandt, J., \& Fürstenberg, F. 1985, A\&A, 143, 72

Krüger, A., Hildebrandt, J., Bogod, V.M., Korzhavin, A.N., Akhmedov, Sh.B., \& Gelfreikh, G.B. 1986, Solar Phys., 105, 111

Krüger, A., Hildebrandt, J., Kliem, B., AuraB, H., Kurths, J., Fomichev, V.V., Chertok, I.M., \& Křivský, L. 1991, Solar Phys., 134, 171

Krüger, A., \& Urpo, S. 1992, in Eruptive Solar Flares, ed. Z. Švestka, B.V. Jackson, \& M.E. Machado (Berlin:Springer), 214

Kuijpers, J. 1975, Solar Phys., 44, 173

Kundu, M.R., \& Alissandrakis, C.E. 1984, Solar Phys., 94, 249

Lang, K.R., Willson, R.F., Smith, K.L., \& Strong, K.T. 1987, ApJ, 322, 1044

Mann, G., \& Baumgärtl, K. 1988, ESA SP-285, 153

Melrose, D.B., Dulk, G.A., \& Smerd, S.F. 1978, A\&A, 66, 315

Newkirk, G. Jr. 1967, ARA\&A, 5, 213

Peterova, N.G., \& Akhmedov, Sh.B. 1973, AZh, 50, 1220

Peterova, N.G., \& Ryabov, B.I. 1981, AZh, 58, 1070

Pikelner, S.B., \& Gintsburg, M.A. 1963, AZh, 40, 842

Roberts, J.A. 1959, Aust. J. Phys., 12, 327

Smerd, S.F., Sheridan, K.V., \& Stewart, R.T. 1975, Astrophys. Lett., 16, 22

Sturrock, P.A. 1961, Nature, 192, 58

Stenflo, J.O. 1973, Solar Phys., 32, 41

Urpo, S., Hildebrandt, J., \& Krüger, A. 1987, Solar Phys., 112, 119

White, S.M., Kundu, M.R., \& Gopalswamy, N. 1991, ApJ, 366, L43

Zheleznyakov, V.V., \& Zlotnik, E.Ya. 1975, Solar Phys., 44, 461 